\title{
The quest to uncover the nature of benzonitrile anion
}

\begin{abstract}
Sahil Gulania, ${ }^{a}$ Thomas-C. Jagau, ${ }^{b}$, Andrei Sanov ${ }^{c}$, and Anna I. Krylov*a
Anionic states of benzonitrile are investigated by high-level electronic structure methods. The calculations using equation-of-motion coupled-cluster theory for electron-attached states confirm earlier conclusions drawn from the photodetachment experiments that the ground state of the anion is the valence ${ }^{2} \mathrm{~B}_{1}$ state, while the dipole bound state lies adiabatically $\sim 0.1 \mathrm{eV}$ above. Inclusion of triple excitations and zero-point vibrational energies is important for recovering relative state ordering. The computed Franck-Condon factors and photodetachment cross-sections further confirm that the observed photodetachment spectrum originates from the valence anion. The valence anion is electronically bound at its equilibrium geometry, but is metastable at the equilibrium geometry of the neutral. The dipole-bound state, which is the only bound anionic state at the neutral geometry, may serve as a gateway state for capturing the electron. Thus, the emerging mechanistic picture entails electron capture via dipole bound state, followed by non-adiabatic relaxation forming valence anion.
\end{abstract}

\section{Introduction}

Benzonitrile $\left(\mathrm{C}_{6} \mathrm{H}_{5} \mathrm{CN}\right)$ has recently become the first aromatic molecule observed in the interstellar medium using a radio telescope $^{1}$. Its detection in the cold-core Tauras Molecular Cloud 1 (TMC-1) has far-reaching implications as it provides a chemical link for unidentified infrared bands in the interstellar medium. These emission features have long been thought to be due to the polycyclic aromatic hydrocarbons $(\mathrm{PAH})^{2}$ and polycyclic aromatic nitrogen heterocycles (PANH) ${ }^{3}$. However, proving the presence of these molecules in the interstellar space has been a challenge for radio-astronomy due to the nonpolar or weakly polar nature of the polycycles combined with the large density of states. Benzonitrile is one of the simplest nitrogen-containing aromatic compounds. As a small molecule with a large dipole moment $(>4$ Debye), it does not have the same detection limitations as the polycycles. The observation of benzonitrile in TMC-1 provides the clearest indication to date that larger PAH and PANH species are likely to be present there as well.

The molecular dipole moment also plays an important role in electron capture and anion formation 49. Among the negative ions previously detected in the interstellar medium 10 , many are carbon-chain species ${ }^{11}-15$, such as $\mathrm{C}_{2 n-1} \mathrm{~N}^{-}$and $\mathrm{C}_{2 n} \mathrm{H}^{-}$. In these systems, electron capture by the neutral molecule may involve doorway dipole-bound states 16 or dipole-stabilized resonances $^{22 \mid 23}$. The large dipole moment of benzonitrile is a predictor of the existence of a dipole-bound state of its anion which may be a precursor for other chemical and photochemical pathways involving negative ions in both the laboratory environments and interstellar space.

The goal of this work is to establish the key electronic properties of benzonitrile, such as its electron affinity (EA) and the

\footnotetext{
a Department of Chemistry, University of Southern California, Los Angeles, California 90089, U.S.A.; E-mail: krylov@usc.edu

${ }^{b}$ Department of Chemistry, University of Munich (LMU), 81377 Munich, Germany

${ }^{c}$ Department of Chemistry and Biochemistry, University of Arizona, Tucson, Arizona 80721, U.S.A.

$\dagger$ Electronic Supplementary Information (ESI) available: relevant Cartesian geometries, basis set details, normal mode analysis.
}

nature of electron binding in its anion. Despite several previous investigations, including a direct photoelectron imaging measurement ${ }^{24}$, the debate about the very nature of benzonitrile anion is still ongoing. The existence of both dipole-bound and valence states of the anion has been predicted $24 \mid 25$. Despite drastically different characters of the respective wave-functions, both types of anions are expected to be weakly bound, with similar electron attachment and detachment energies, making the assignment and interpretation of experimental data a delicate task.

The first measurement of the EA of benzonitrile dates back to $1975^{26}$, when it was indirectly determined to be $0.256(17)$ eV. Another, also indirect, measurement ${ }^{27}$ based on electron capture detection in 1983 yielded a value with a significantly larger uncertainty, $0.26(10) \mathrm{eV}$, nonetheless in perfect agreement with the earlier determination. Subsequent 1992 experiment indicated that the ground-state anion of benzonitrile could not be observed by electron transmission spectroscopy and hence it was concluded that the anion is bound by a few tenths of an electron-volt 28 .

The 2015 photoelectron imaging experiment by Sanov and coworkers 24 yielded the photoelectron spectra and angular distributions for the bare and microsolvated benzonitrile anion. Fig. 1 shows the photoelectron spectra of $\mathrm{C}_{6} \mathrm{H}_{5} \mathrm{CN}^{-}$and $\mathrm{C}_{6} \mathrm{H}_{5} \mathrm{CN}^{-} \cdot \mathrm{H}_{2} \mathrm{O}$ obtained at two different photon energies. The authors reported vertical detachment energy (VDE) of bare $\mathrm{C}_{6} \mathrm{H}_{5} \mathrm{CN}^{-}$of $0.058(5)$ $\mathrm{eV}$, while in the $\mathrm{C}_{6} \mathrm{H}_{5} \mathrm{CN}^{-} \cdot \mathrm{H}_{2} \mathrm{O}$ cluster the band was blue-shifted by $0.32 \mathrm{eV}$. The spectra were assigned to the valence anion (VA) of benzonitrile, although a contribution of the dipole-bound state (DBS) could not be ruled out. The authors also calculated the EAs and VDEs for $\mathrm{C}_{6} \mathrm{H}_{5} \mathrm{CN}^{-}$and $\mathrm{C}_{6} \mathrm{H}_{5} \mathrm{CN}^{-} \cdot \mathrm{H}_{2} \mathrm{O}$ using equationof-motion coupled-cluster (EOM-CC) methods 29 . Of particular note, the computed VDE of $\mathrm{C}_{6} \mathrm{H}_{5} \mathrm{CN}^{-}(0.047 \mathrm{eV})$ was in good agreement with the $0.058(5) \mathrm{eV}$ experimental value. The calculations were carried out for the valence state of the benzonitrile anion in which the extra electron occupied a $\pi^{*}$-like $\left(b_{1}\right)$ orbital and the target photodetachment transition corresponded to electron removal from the singly occupied HOMO of the anion. The assignment of the experimental spectrum to the VA of benzonitrile took into account the good agreement between the 

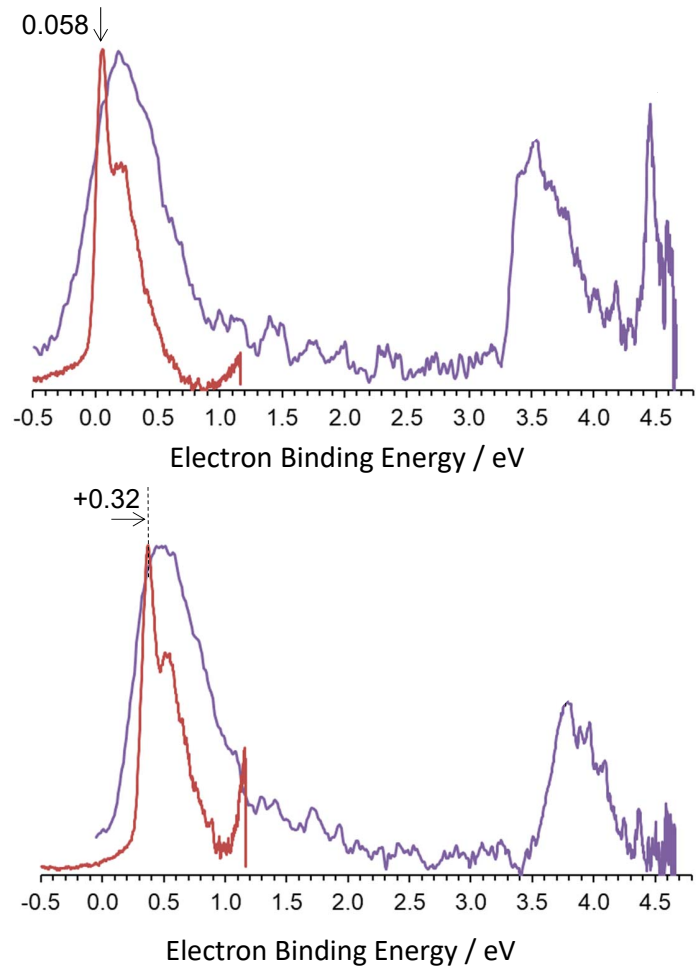

Fig. 1 Photoelectron spectra of $\mathrm{C}_{6} \mathrm{H}_{5} \mathrm{CN}^{-}$(top) and $\mathrm{C}_{6} \mathrm{H}_{5} \mathrm{CN}^{-} \cdot \mathrm{H}_{2} \mathrm{O}$ (bottom) obtained with two different energy photons. Red and blue lines correspond to the spectra obtained with $1.165 \mathrm{eV}$ and $4.661 \mathrm{eV}$ photons, respectively. Reproduced with permission from Ref. 24

computed and experimental VDE values. It was additionally supported by the analysis of the photoelectron angular distributions, the Franck-Condon simulation of the low-energy photodetachment band, and the behavior of the band under the microsolvation conditions. However, while concluding that the experimental results could be completely explained by the VA structure of benzonitrile, the authors did not explicitly analyze the DBS.

In a subsequent computational study 25 , Adamowicz and coworkers examined the structures and energetics of both the VA and DBS of benzonitrile using the $\operatorname{CCSD}(\mathrm{T})$ method $\left(\mathrm{CCSD}^{32}\right.$ with perturbative account of triple excitations ${ }^{33}$ ). They reported $^{25}$ the VDE of the DBS of benzonitrile to be $0.019 \mathrm{eV}$, while claiming that the VA is adiabatically unbound. Although the above VDE did not agree well with the published photoelectron spectra, the authors nonetheless suggested that the experimental spectra of Sanov and co-workers should be attributed to the DBS, rather than the VA of benzonitrile.

In the present study, we resolve the ambiguity created by the above conflicting conclusions. We report high-level calculations showing that the photodetachment transitions observed by Sanov and co-workers are indeed attributed to the valence anion, but also discuss the possibility that the DBS may serve as a doorway state in the formation of the VA. This route is easily accessible in benzonitrile and therefore future detection of its anion in ISM should be anticipated.

\section{Theoretical methods}

The EOM-CC approach $29|31 / 34| 41$ provides an efficient and robust framework for computing a variety of electronic states in closedand open-shell species. Different variants of EOM-CC enable access to different types of target electronic states, such as electronically excited, electron attached or ionized states. The EOM-CC wave-function has the following form:

$$
|\Psi\rangle=R e^{T}\left|\Phi_{0}\right\rangle
$$

where the linear EOM operator $R$ acts on the reference CC wavefunction, $e^{T}\left|\Phi_{0}\right\rangle$. The operator $T$ is an excitation operator satisfying the CC equations for the reference state

$$
\left\langle\Phi_{\mu}|\bar{H}| \Phi_{0}\right\rangle=0
$$

where $\Phi_{\mu}$ are the $\mu$-tuply excited determinants and $\bar{H}=e^{-T} H e^{T}$. In EOM-CCSD, the CC and EOM operator are truncated as follows $32 \mid 38$ :

$$
T \approx T_{1}+T_{2}, \quad R \approx R_{0}+R_{1}+R_{2},
$$

where $T_{1}$ and $T_{2}$ are single and double excitation operators, 1hole-1-particle $(1 h 1 p)$ and 2-holes-2-particles $(2 h 2 p)$ :

$$
T_{1}=\sum_{i a} t_{i}^{a} a^{\dagger} i, \quad T_{2}=\frac{1}{4} \sum_{i j a b} t_{i j}^{a b} a^{\dagger} b^{\dagger} j i .
$$

Different variants 29 of EOM-CC are defined by different choices of the reference state and the type of EOM operators $R$. For example, by choosing the reference as a neutral state and $R_{1}$ and $R_{2}$ as $1 h$ and $2 h 1 p$ operators, one can describe ground and excited states of the cation. This strategy was used by Sanov and co-workers ${ }^{24}$, who computed VDE by EOM-IP-CCSD from the open-shell VA state of benzonitrile. A more balanced treatment of open-shell anionic states is afforded by the EOM-EA-CCSD variant ${ }^{42}$ in which the reference is a neutral closed-shell state and $R_{1}$ and $R_{2}$ are of $1 p$ and $1 h 2 p$ type, as illustrated in Fig. 2.

$$
R_{1}=\sum_{i} r^{a} a^{\dagger}, \quad R_{2}=\frac{1}{2} \sum_{i a b} r_{i}^{a b} a^{\dagger} b^{\dagger} i
$$

Here we characterize the VA and DBS of benzonitrile by EOMEA-CCSD starting from the ground state of the neutral benzonitrile; in these calculations VDE is obtained as the energy difference between the EOM-EA-CCSD and CCSD states. The accuracy of EOM-CC can be systematically improved by including higherlevel of excitations in $T$ and $R$, up to the exact limit. Here we account for the effect of triple excitations by using perturbative correction, i.e., the EOM-EA-CCSD(T) (a)* method $43 / 44$.

The EOM amplitudes and the corresponding energies are found by diagonalizing the similarity transformed Hamiltonian, $\bar{H}$. Since $\bar{H}$ is a non Hermitian operator, its left and right eigenstates are not identical but can be chosen to form a biorthonormal set.

$$
\begin{gathered}
\bar{H} R\left|\Phi_{0}\right\rangle=E R\left|\Phi_{0}\right\rangle, \\
\left\langle\Phi_{0}\right| L \bar{H}=\left\langle\Phi_{0}\right| L E,
\end{gathered}
$$




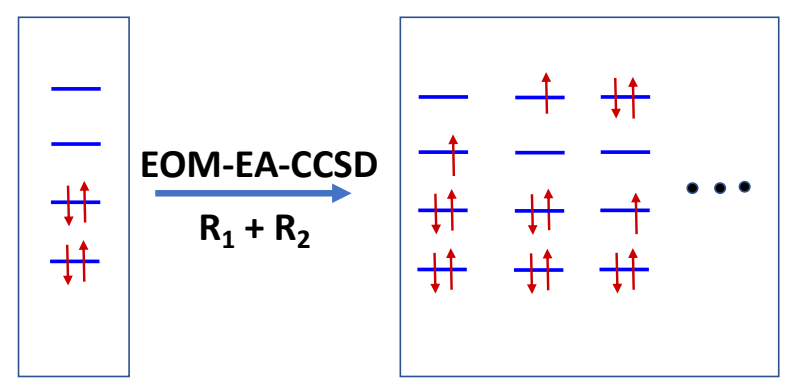

Fig. 2 EOM-EA target configurations generated from a closed-shell reference state. The first two configurations are $1 p$ ones and the third one is $2 p 1 h$.

$$
\left\langle\Phi_{0}\left|L^{M} R^{N}\right| \Phi_{0}\right\rangle=\delta_{M N},
$$

where $M$ and $N$ denote the $M^{\text {th }}$ and $N^{t h}$ EOM states and

$$
L=L_{1}+L_{2}=\sum_{a} l_{a} a+\frac{1}{2} \sum_{i a b} l_{a b}^{i} i^{\dagger} b a .
$$

The left and right amplitudes are found by diagonalizing the corresponding matrix representation of $\bar{H}$. For energy calculations, right eigenstates are sufficient, but for property calculations, such as Dyson orbitals 45 , both left and right eigenstates need to be computed.

Dyson orbitals are reduced quantities defined as the overlap between an initial $N$-electron and final $N$-1-electron states:

$$
\phi_{i f}^{\text {Dyson }}\left(x_{1}\right)=\sqrt{N} \int \Psi_{i}^{N}\left(x_{1}, x_{2}, \ldots, x_{N}\right) \Psi_{f}^{N-1}\left(x_{2}, \ldots, x_{N}\right) d x_{2} \ldots d x_{N} .
$$

Because Dyson orbitals enter the expressions of various experimental observables, such as photoionization/photodetachment cross sections $46 / 47$, they can be interpreted as correlated states of the ejected/attached electron. Thus, Dyson orbitals provide a basis for a rigorous extension of molecular orbital theory to manybody correlated wave functions ${ }^{45 / 48 / 49}$. Here we compute Dyson orbitals using the CCSD (as $\Psi_{f}^{N-1}$ ) and EOM-EA-CCSD (as $\Psi_{i}^{N}$ ) wave functions of the neutral and of the anion, respectively.

\section{Computation details}

As outlined above, we characterized valence and dipole-bound states of the benzonitrile anion by EOM-EA-CCSD using the neutral CCSD reference. Core electrons were frozen in all correlated calculations. Our primary focus is on the relative energetics of the neutral, VA, and DBS. For negative ions, VDE is defined as energy gap between the ground-state energy of the anion and the corresponding neutral molecule, both at the equilibrium geometry of the anion $\left(\mathbf{R}_{A}\right)$ :

$$
\mathrm{VDE}=E_{N}\left(\mathbf{R}_{A}\right)-E_{A}\left(\mathbf{R}_{A}\right)
$$

Analogously, vertical attachment energy (VAE) is computed at the equilibrium geometry of the ground-state neutral molecule $\left(\mathbf{R}_{N}\right)$ :

$$
\mathrm{VAE}=E_{A}\left(\mathbf{R}_{N}\right)-E_{N}\left(\mathbf{R}_{N}\right)
$$

The electronic part of the adiabatic electron affinity $\left(\mathrm{AEA}_{e e}\right)$ is defined as the energy difference between the ground-state energy of the anion at its equilibrium geometry and the ground-state energy of the neutral at its equilibrium geometry:

$$
\mathrm{AEA}_{e e}=E_{N}\left(\mathbf{R}_{N}\right)-E_{A}\left(\mathbf{R}_{A}\right) .
$$

To obtain the relative ordering of the anionic states and to compare with the experimental photodetachment onset, we also computed AEA, which includes zero-point energy (ZPE) differences between the anion and the neutral states:

$$
\mathrm{AEA}=\mathrm{AEA}_{e e}+\Delta \mathrm{ZPE}
$$

In calculations of energetics, we used the geometry of the neutral and VA states optimized by CCSD/aug-cc-pVTZ and EOMEA-CCSD/aug-cc-pVTZ, respectively. Both structures are of $\mathrm{C}_{2 v}$ symmetry. As is well documented in the literature ${ }^{4 / 5}$, the shape of potential energy surfaces of dipole-bound anions are very similar to the respective structures of the neutral species because the extra electron resides largely outside the molecular core. Thus, the energies of DBS were computed at the geometries of the neutral benzonitrile. Triples corrections to the VDE and AEA of the VA were computed with EOM-EA-CCSD(T) (a)* method ${ }^{43 / 44}$ using aug-cc-pVTZ. We assume that the effect of triples cancels out for the DBS, because the unpaired electron does not participate in the bonding.

Because of their structural similarity, we also expect vibrational frequencies of the neutral and DBS to be similar, giving rise to $\triangle \mathrm{ZPE} \approx 0$. ZPEs of the neutral and the VA states were computed with CCSD and EOM-EA-CCSD using aug-cc-pVDZ and resolution-of-the-identity (RI) approximation $50 \mid 51$ with the matching basis set (ri-aug-cc-pVDZ), at the geometries optimized at the same level of theory. The computed structures and normal modes were used to compute the Franck-Condon factors within parallel-mode double-harmonic approximation using the ezSpectrum software 52 . To further elucidate putative contributions of the DBS to the spectra, we computed photoelectron cross sections using EOM-EA-CCSD Dyson orbitals and the ezDyson software 53 .

To correctly describe DBS, large basis sets with additional sets of diffuse functions are needed. We used the aug-cc-pVTZ basis augmented with several extra sets of diffuse functions added to each atom, with the exponents obtained following the same procedure as in our previous studies 23|54|57; the details are provided in the SI. Our preliminary calculations monitoring the convergence of the VAE of the DBS showed that the results converge with the aug-cc-pVTZ+6s3p(3s) basis. Here, "6s3p" refers to the additional diffuse functions placed at the heavy atoms and " $(3 \mathrm{~s})$ " to those placed at the hydrogen atoms. Below we report the energetics of the bare benzonitrile anion obtained with augcc-pVTZ $+6 s 3 p(3 s)$. For the $\mathrm{C}_{6} \mathrm{H}_{5} \mathrm{CN}^{-} \cdot \mathrm{H}_{2} \mathrm{O}$ complex, the VEA of the DBS converged with the aug-cc-pVTZ $+4 s 4 p(4 s)$ basis. Thus, for the benzonitrile-water complex, we report energetics obtained with aug-cc-pVTZ+4s4p(4s).

All electronic structure calculations were performed using the Q-Chem package 58 59 . Below we report symmetry labels using Mulliken's convention 60 . Basis sets, relevant Cartesian coordinates, and vibrational frequencies are given in the SI. 


\section{Results and discussion}

\subsection{The benzonitrile anion}

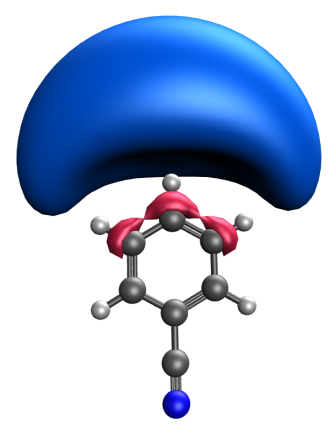

DBS $\left({ }^{2} A_{1}\right)$

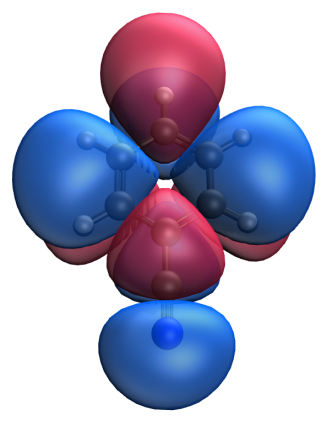

VA $\left({ }^{2} B_{1}\right)$
Fig. 3 Dyson orbitals for the two lowest electronic states of the benzonitrile anion, ${ }^{2} \mathrm{~A}_{1}$ and ${ }^{2} \mathrm{~B}_{1}$, computed at the respective optimized geometries. Isovalue 0.007 .

Fig. 3 shows Dyson orbitals of the two lowest states of the benzonitrile anion, ${ }^{2} \mathrm{~A}_{1}$ and ${ }^{2} \mathrm{~B}_{1}$. The shape of the orbitals identifies the former as DBS and the latter as VA. In the VA state, the unpaired electron resides on a relatively compact $\pi^{*}$-like orbital of $b_{1}$ symmetry, giving rise to the ${ }^{2} \mathrm{~B}_{1}$ state. The Dyson orbital for the DBS is a diffuse $s$-like orbital located on the opposite end of the cyano-group, giving rise to the ${ }^{2} \mathrm{~A}_{1}$ state. The DBS is supported by the large dipole moment of benzonitrile, 4.57 Debye (CCSD/aug-cc-pVTZ).

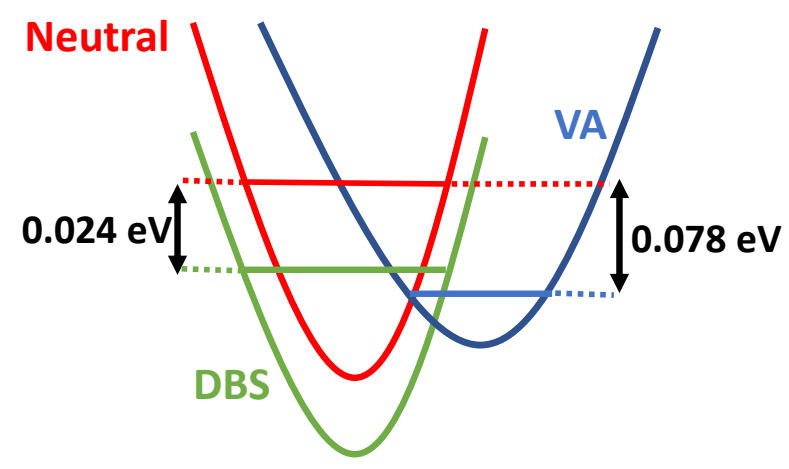

Fig. 4 Schematic representation of the energy levels of the neutral, valence and dipole-bound anionic states of benzonitrile (see text). Note that the VA adiabatically drops below the neutral and the DBS due to zero-point energy.

Fig. 4 shows the schematic energy diagram of the neutral benzonitrile and the two anionic states. The present EOM-EACCSD calculations reveal that at the neutral's equilibrium geometry $\left(\mathbf{R}_{N}\right)$, the VA state is electronically unbound, and the only bound anionic state is the DBS, with VEA of $0.0240 \mathrm{eV}$. This finding is consistent with the corresponding results of Adamowicz and co-workers ${ }^{25}$, who reported VDE of the DBS to be $0.019 \mathrm{eV}$ with $\operatorname{CCSD}(\mathrm{T})$. However, at the optimized geometry of the valence an- ion, both the DBS and VA are (vertically) bound by $0.0264 \mathrm{eV}$ and $0.0639 \mathrm{eV}$, respectively. The latter value is in excellent agreement with the experimental VDE of $0.058(5) \mathrm{eV}$, previously assigned to the $\mathrm{VA}^{24}$. We note that this value is a significant improvement over the corresponding EOM-IP-CCSD value of $0.047 \mathrm{eV}$, which was obtained using a less balanced protocol based on the openshell anionic reference and a smaller basis set 24 .

Considering only the electronic energies (computed with EOMEA-CCSD), the DBS minimum is $0.139 \mathrm{eV}$ below the minimum of the VA and the VA is adiabatically unbound (as indicated by the negative $\mathrm{AEA}_{e e}$ ). However, the ZPE correction makes the VA adiabatically bound by $0.0106 \mathrm{eV}$. A relatively large effect of $\triangle \mathrm{ZPE}$ favoring the VA can be easily rationalized by the shapes of the respective Dyson orbitals (Fig. 3): because the electron is attached to the $\pi^{*}$ orbital, the vibrational modes of the anion become softer, thus lowering the magnitude of ZPE relative to the neutral. The mode that is most affected by electron attachment is the butterfly mode. The frequency of this mode softens by $\sim 170$ $\mathrm{cm}^{-1}$ upon electron attachment, which is clearly illustrated in Fig. 5 by the reduction of the curvature of the potential energy profile. The frequencies are given in Table SI in the SI.

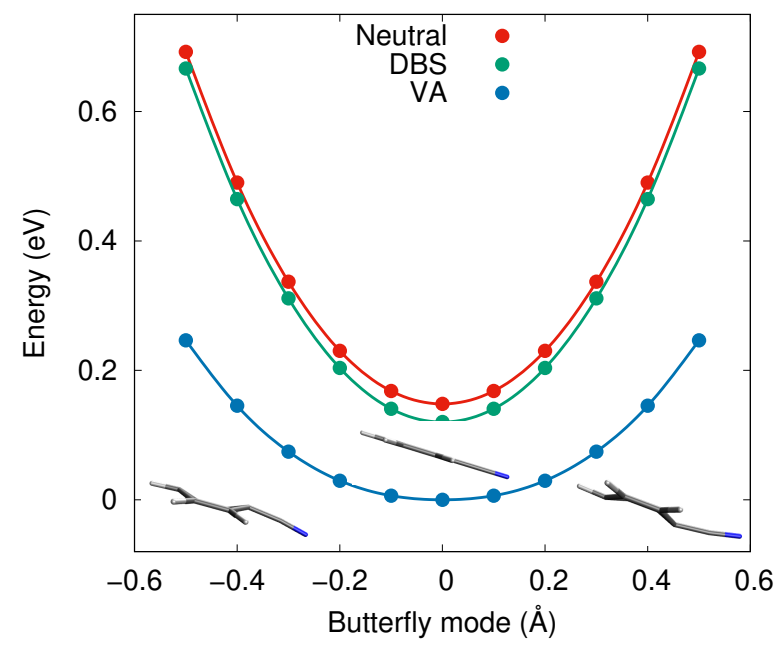

Fig. 5 Potential energy curves along the butterfly mode for VA, DBS, and the neutral, showing the relaxation of VA. Energies along the scan are computed with EOM-EA-CCSD/aug-cc-pVDZ+6s3p(3s). The scan was generated by taking the displacement along the butterfly normal mode of the anion (mode \#2 of $209.18 \mathrm{~cm}^{-1}$ ).

The inclusion of perturbative triple excitations increases the attachment energy of the VA by $0.0677 \mathrm{eV}$. Thus, the state ordering is reversed and the VA becomes adiabatically more stable than the DBS when both triple excitations and ZPE are taken into account. Table 1 summarizes the key energetics and Fig. 4 presents the results graphically.

Fig. 4. which shows the relevant energy levels of benzonitrile and its anion, highlights that the VA is bound by $0.078 \mathrm{eV}$ relative to the ground state of the neutral and is $0.054 \mathrm{eV}$ more stable adiabatically than the DBS. However, at the geometry of the neutral, the VA is above the DBS and is electronically unbound. Thus, the DBS may act as a doorway for the VA formation. In this mecha- 
Table 1 Attachment and detachment energies (in eV) for valence $\left({ }^{2} B_{1}\right)$ and dipole-bound $\left({ }^{2} A_{1}\right)$ anion of benzonitrile.

\begin{tabular}{|lcccccc|}
\hline State & $\mathrm{VAE}^{a}$ & $\mathrm{VDE}^{a}$ & $\mathrm{AEA}_{e e}^{a}$ & $\Delta \mathrm{ZPE}^{b}$ & $\Delta(\mathrm{T})^{c}$ & $\mathrm{AEA}^{d}$ \\
\hline${ }^{2} \mathrm{~A}_{1}$ & -0.0240 & - & 0.0240 & $\sim 0$ & $\sim 0$ & 0.024 \\
${ }^{2} \mathrm{~B}_{1}$ & $\mathrm{NB}^{e}$ & 0.0639 & -0.1150 & 0.1256 & 0.0677 & 0.078 \\
\hline
\end{tabular}

${ }^{a}$ EOM-EA-CCSD/aug-cc-pVTZ+6s3p(3s).

${ }^{b}$ RI-CCSD/RI-EOM-EA-CCSD and aug-cc-pVDZ for the VA.

${ }^{c}$ EOM-EA-CCSD(T) (a)*/aug-cc-pVTZ for the VA.

${ }^{d}$ AEA including $\triangle \mathrm{ZPE}$ and triples correction.

${ }^{e}$ Electronically not bound.

nism, the electron is first captured by benzonitrile at its neutral geometry, forming the DBS anion, followed by a non-adiabatic transition to the more stable VA state.

\subsection{The $\mathrm{C}_{6} \mathrm{H}_{5} \mathrm{CN}^{-} \cdot \mathrm{H}_{2} \mathrm{O}$ complex}

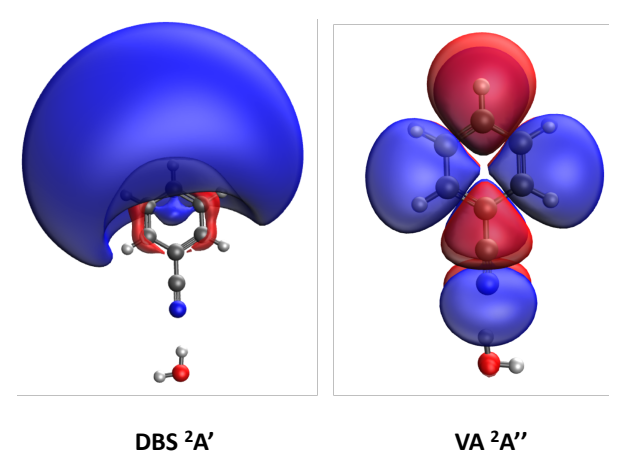

Fig. 6 Dyson orbitals for the two lowest electronic states of the $\mathrm{C}_{6} \mathrm{H}_{5} \mathrm{CN}^{-} \cdot \mathrm{H}_{2} \mathrm{O}$ complex, ${ }^{2} \mathrm{~A}^{\prime}$ and ${ }^{2} \mathrm{~A}^{\prime \prime}$, computed at the respective optimized geometries. Isovalue 0.007 .

In the $\mathrm{C}_{6} \mathrm{H}_{5} \mathrm{CN}^{-} \cdot \mathrm{H}_{2} \mathrm{O}$ complex, the water molecule forms a hydrogen bond with the cyano group, only weekly perturbing the electronic structure of the benzonitrile core. The electronic states of the anion appear to be very similar to those of the bare benzonitrile, as one can clearly see from the Dyson orbitals shown in Fig. 6. However, microsolvation affects the energetics of the states. At the neutral's geometry, the $\operatorname{DBS}\left({ }^{1} \mathrm{~A}^{\prime}\right)$ and $\operatorname{VA}\left({ }^{1} \mathrm{~A}^{\prime \prime}\right)$ are vertically bound by $0.0661 \mathrm{eV}$ and $0.0535 \mathrm{eV}$, respectively. The detachment energy for VA increases approximately tenfold, up to $0.4566 \mathrm{eV}$ at the optimized geometry of the VA. Adiabatically, the DBS and VA are bound by 0.0661 and $0.169 \mathrm{eV}$, respectively.

Fig. 7 shows the relevant state ordering in this complex. Here, the ZPE also makes the VA more bound, but the effect is small, relative to the VDE itself. The computed VDE agrees with the experimental electron detachment energies, confirming that electron detachment happens from the VA. One crucial experimental observation is that the detachment energy increases by $0.32 \mathrm{eV}$ upon addition of water relative to the bare benzonitrile. This value is in good agreement with the $0.39 \mathrm{eV}$ increase in VDE predicted by theory.

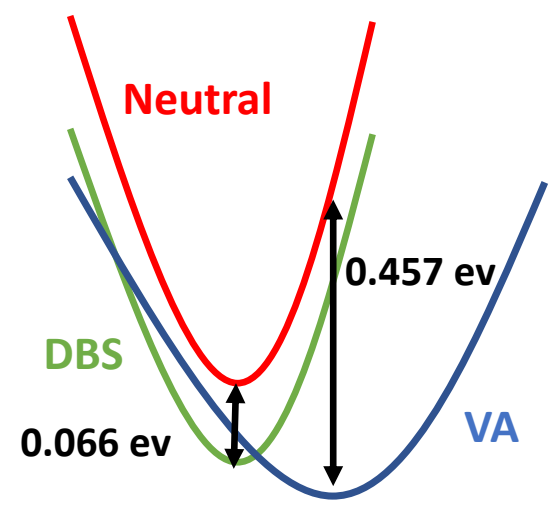

Fig. 7 Schematic representation of the neutral, valence anion, and dipolebound states of the benzonitrile-water complex.

Table 2 Attachment and detachment energies (in eV) for the valence $\left({ }^{1} A^{\prime \prime}\right)$ and dipole-bound $\left({ }^{2} A^{\prime}\right)$ states of the benzonitrile-water complex.

\begin{tabular}{|l|c|c|c|}
\hline State & $\mathrm{VAE}^{a}$ & $\mathrm{VDE}^{a}$ & $\mathrm{AEA}_{e e}^{a}$ \\
\hline${ }^{2} \mathrm{~A}^{\prime}$ & -0.0661 & - & 0.0661 \\
${ }^{2} \mathrm{~A}^{\prime \prime}$ & -0.0535 & 0.4566 & 0.169 \\
\hline
\end{tabular}

${ }^{a}$ EOM-EA-CCSD/aug-cc-pVTZ+4s4p(4s)

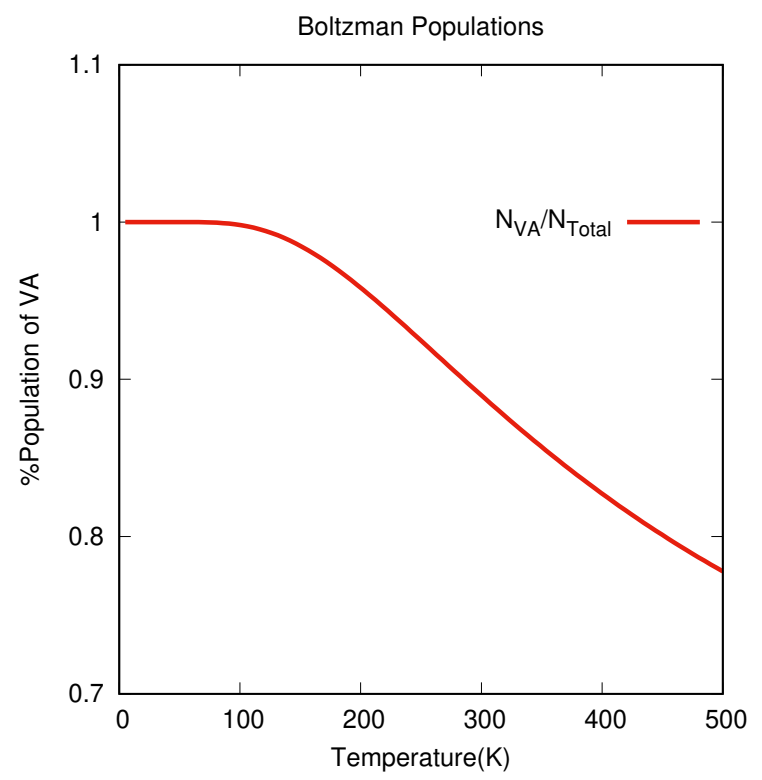

Fig. 8 Ratio of the Boltzmann population of VA $\left(N_{V A}\right)$ relative to the total population $\left(N_{\text {total }}\right)$ as a function of temperature.

\subsection{Photodetachment spectra}

The computed energetics of the bare benzonitrile suggest that the VA species are dominant even at room temperature (300 K), as shown in Fig. 8 The much larger gap between the VA and DBS in the microsolvated benzonitrile makes the presence of the DBS in $\mathrm{C}_{6} \mathrm{H}_{5} \mathrm{CN}^{-} \cdot \mathrm{H}_{2} \mathrm{O}$ highly improbable. In this section, we analyze Franck-Condon factors and photodetachment cross sections for the VA and DBS, to further confirm our assignment.

Using the geometries of the anion and neutral forms of ben- 


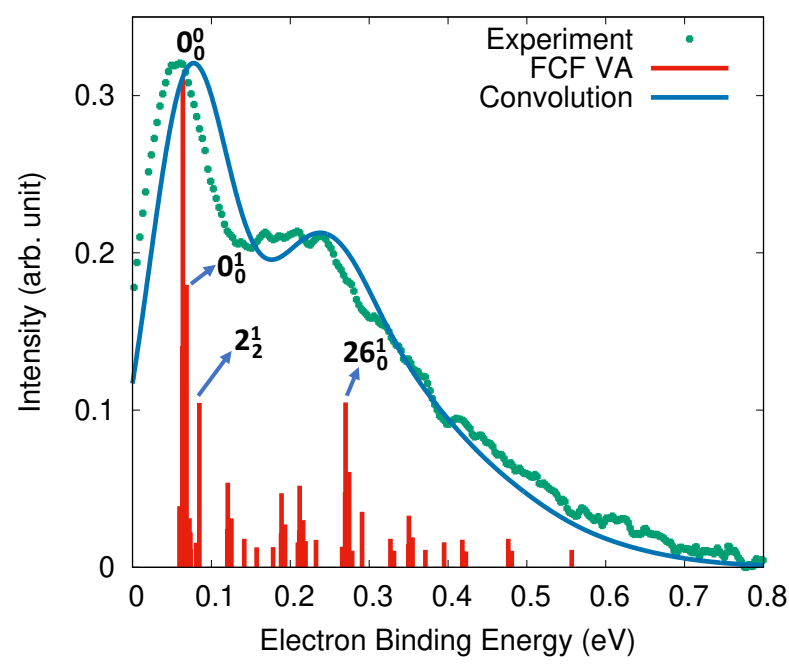

Fig. 9 Computed photoelectron spectrum for the VA and the experimental spectrum obtained by Sanov and co-workers 24 . In the computed spectrum, the Franck-Condon factors were convoluted with gaussians of width $0.05 \mathrm{eV}$.
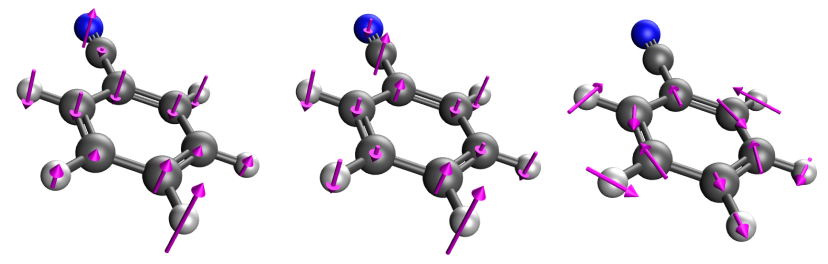

Fig. 10 Franck-Condon active modes: \#0 (out-of-plane), \#2 (butterfly), and \#26 (ring breathing).

zonitrile, we computed the Franck-Condon factors for the photodetachment from the VA state and compared them with the experimental spectrum in Fig. 9 The spectra show broad vibrational progression. The analysis of the computed Franck-Condion factors (Table 3) reveals three dominant modes: Butterfly mode (\#2), ring-breathing mode (\#26), and another soft out-of-plane mode (\#0). The frequencies are shown in Table SI of the SI and the modes are shown in Fig. 10. The character of the FranckCondon modes is consistent with the detachment from an antibonding $\pi^{*}$ orbital (Fig. 3, right panel).

As the formation of the DBS of the anion does not lead to a significant change in geometry, the detachment from DBS would result in the sharp 00 transition with no vibrational structure. Thus, the observed vibrational structure in the measured photodetachment spectrum also supports the conclusion that the observed photoelectron spectra must be attributed to the VA rather than the DBS.

Extending the same argument to the $\mathrm{C}_{6} \mathrm{H}_{5} \mathrm{CN}^{-} \cdot \mathrm{H}_{2} \mathrm{O}$ complex, the VA is expected to show a similar Franck-Condon progression in the photoelectron spectra. In the experiment with $1.165 \mathrm{eV}$ photons, both $\mathrm{C}_{6} \mathrm{H}_{5} \mathrm{CN}^{-}$and $\mathrm{C}_{6} \mathrm{H}_{5} \mathrm{CN}^{-} \cdot \mathrm{H}_{2} \mathrm{O}$ indeed show similar Franck-Condon envelops. This observation strongly suggests that the observed photoelectron spectra for both $\mathrm{C}_{6} \mathrm{H}_{5} \mathrm{CN}^{-}$and $\mathrm{C}_{6} \mathrm{H}_{5} \mathrm{CN}^{-} \cdot \mathrm{H}_{2} \mathrm{O}$ correspond to the detachment from VA.
Table 3 Peak positions and assignments for the photoelectron spectrum of $\mathrm{C}_{6} \mathrm{H}_{5} \mathrm{CN}^{-}\left({ }^{1} \mathrm{~A}_{1} \leftarrow{ }^{2} \mathrm{~B}_{1}\right)$.

\begin{tabular}{|c|c|c|}
\hline Energy (eV) & Intensity (arb. units) & Vibration \\
\hline 0.0634 & 0.1406 & $1_{1}^{1}$ \\
\hline 0.0639 & 0.3106 & $0_{0}^{0}$ \\
\hline 0.0684 & 0.1797 & $0_{0}^{1}$ \\
\hline 0.0847 & 0.1046 & $2_{2}^{1}$ \\
\hline 1.2701 & 0.1049 & $26_{0}^{1}$ \\
\hline
\end{tabular}

To assess whether possible contributions from the DBS to photoelectron spectra are not seen in the experiment due to potentially low cross section, we also computed absolute photodetachment cross sections using Dyson orbitals and ezDyson software 53 . Different orbital characters of DBS and VA result in different trends in the computed cross sections. The absolute cross sections for the two anionic states are shown in Fig. S2 of SI. Fig. 11]shows the dependence of ratio of the two cross sections as a function on the photon energy. The results reveal that at low photon energy the DBS photodetachment cross section is 100 times higher than that of VA. At the $1.165 \mathrm{eV}$ photon energy, corresponding to the experimental spectrum shown in Fig. 9, our calculations predict $\sigma_{D B S} / \sigma_{V A}=4.65$ (see the inset Fig. 11). Taking this ratio value and estimating the relative intensity of DBS photodetachment at this energy as

$$
\mathbf{I}_{D B S}=\mathbf{I}_{V A} \times \frac{\sigma_{D B S}}{\sigma_{V A}}
$$

one can compute the Franck-Condon spectra for an arbitrary mixture of DBS and VA. Fig. 11 shows such a spectrum for an equal mixture of the two anionic states. The sharp threshold peak due to the DBS has a very high relative intensity, dwarfing the FranckCondon envelope due to the VA. This shows that if the population of the DBS were significant, the first sharp peak in the photoelectron spectrum would be attributed to DBS. However, the very similar first peak in the $\mathrm{C}_{6} \mathrm{H}_{5} \mathrm{CN}^{-} \cdot \mathrm{H}_{2} \mathrm{O}$ spectrum (Fig. 1, bottom) should definitely be attributed to VA, based on the energetics shown in Fig. 77. Disregarding the $0.32 \mathrm{eV}$ band shift, the $\mathrm{C}_{6} \mathrm{H}_{5} \mathrm{CN}^{-}$and $\mathrm{C}_{6} \mathrm{H}_{5} \mathrm{CN}^{-} \cdot \mathrm{H}_{2} \mathrm{O}$ spectra in Fig. 1 are indeed very similar, strongly suggesting that the electronic character of the core anion should be the same in both cases. This comparison supports the argument that the first peak observed in the experimental spectra of both $\mathrm{C}_{6} \mathrm{H}_{5} \mathrm{CN}^{-}$and $\mathrm{C}_{6} \mathrm{H}_{5} \mathrm{CN}^{-} \cdot \mathrm{H}_{2} \mathrm{O}$ originates from the VA. If the DBS were involved, it would contributed more prominently (with a sharp threshold peak) to the spectrum of the unsolvated anion, compared to the micro-hydrated species, overpowering the Franck-Condon features from the VA.

A similar interplay between the VA and DBS was observed in uracil 61/62. An experimental study by Bowen et. al ${ }^{63}$ was able to distinguish between the DBS and VA in uracil. The photoelectron spectrum of the uracil anion shows a strong narrow single peak at $93 \mathrm{meV}$. The absence of the Franck-Condon progression indicated negligible structural changes from the anion to the neutral, which is characteristic of DBS. Using argon and krypton as solvents did not affect the narrow peak, but when xenon was used as solvent, the spectra changed dramatically. This behavior was attributed 

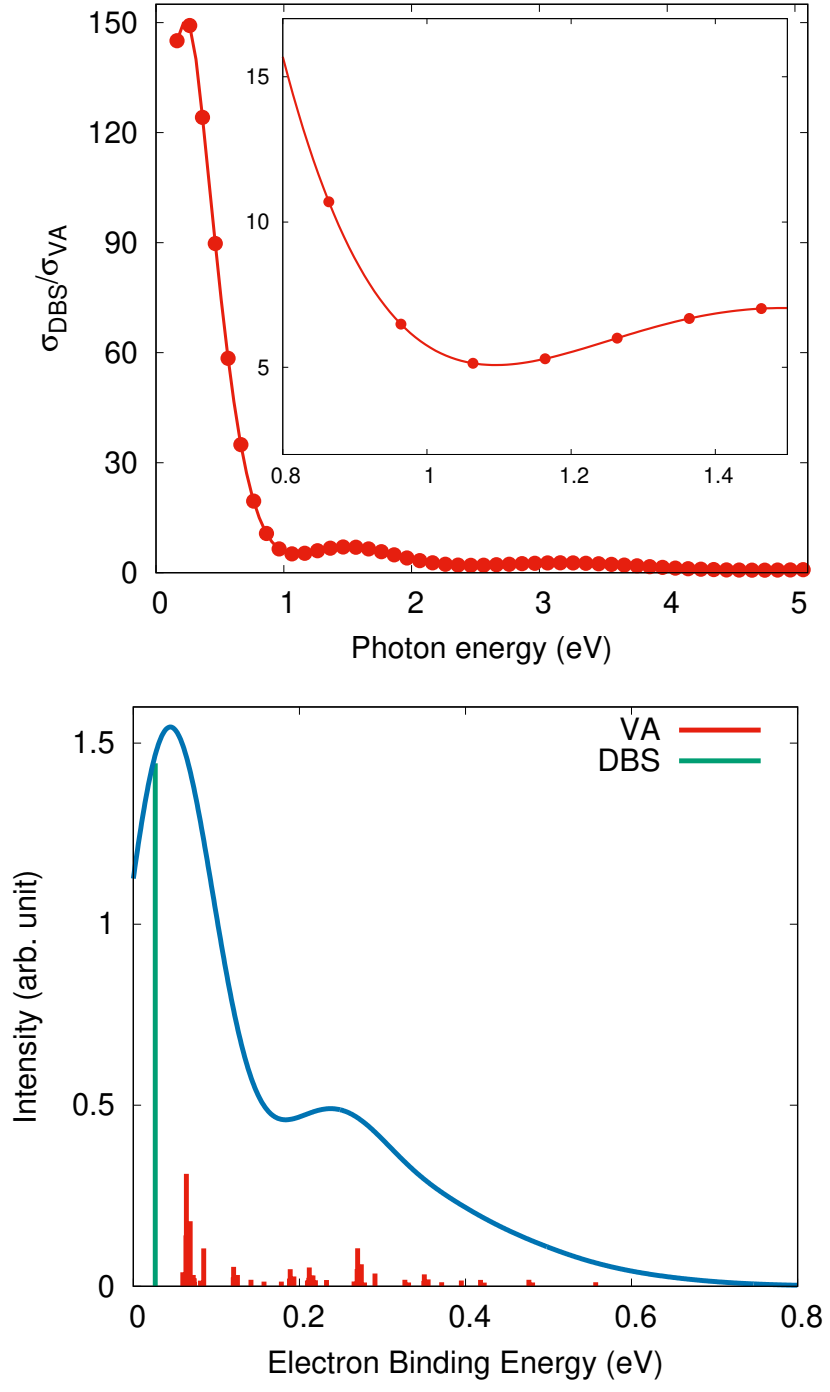

Fig. 11 Top: Ratio of the cross sections (DBS versus VA) for electron detachment from the benzonitrile anion. Bottom: Convolution of the Franck-Condon factors using Eq. (15) with gaussian (width=0.05 eV) and assuming equal populations of the DBS and VA.

to the polarization effect of xenon, which resulted in breaking the symmetry of the molecule and stabilizing the VA. Using more polar solvent such as $\mathrm{H}_{2} \mathrm{O}$ resulted in complete disappearance of the DBS peak.

The behavior of the benzonitrile anion differs from that of uracil in several respects. First, the Franck-Condon features are observed in the photoelectron spectrum with no solvent present. Second, no additional sharp peaks, which could be attributed to the DBS, are seen in the photodetachment of the unsolvated anion, as compared to the micro-solvated species. Third, the VA of benzonitrile is adiabatically more stable than the DBS. Thus, the DBS may act as a doorway to capture the electron, subsequently transferring the population into the VA upon the collisional relaxation of the anion. Since the cross sections for photodetachment are equal to the cross sections of the reverse process, radiative electron attachment, the large value of the cross section for DBS further supports that electron capture directly into this state may be efficient. Given the computed structures and frequencies, it is likely that the butterfly mode drives the relaxation of the DBS into the VA.

\section{Conclusion}

CCSD and EOM-EA-CCSD calculations of the electronic states of benzonitrile and its anion reveal that although, in terms of electronic energies the VA is adiabatically above the neutral state, the respective ZPE-corrected levels reverse the state ordering, making the VA bound. The inclusion of triples corrections further stabilizes the VA state relative to the neutral and DBS. The computed energetics are in excellent agreement with the experimental values reported in earlier work 24 . The computed Franck-Condon factors provide further support to the assignment of the ground state of the benzonitrile anion as the valence-bound anion, ${ }^{2} \mathrm{~B}_{1}$, rather than dipole-bound state $\left({ }^{2} \mathrm{~A}_{1}\right)$, as was claimed by Adamowicz and co-workers 25 . The DBS ${ }^{2} \mathrm{~A}_{1}$, which is $0.054 \mathrm{eV}$ above the VA (adiabatically) and is electronically bound at the equilibrium geometry of the neutral, is likely serving as a doorway state for electron capture. The calculations on benzonitrile solvated with one water molecule show that the VA is stabilized further and becomes bound even at the structure of the neutral complex. The calculations of photoelectron spectra show similar FranckCondon envelops, blue-shifted by $0.39 \mathrm{eV}$ with respect to the bare benzonitrile, also in excellent agreement with the experimental findings $(0.32 \mathrm{eV})$ of Sanov and co-workers 24 . As was pointed out in the original experimental study 24 , the similarity in the vibrational structure of the photodetachment spectra of the bare and microsolvated benzonitrile provides additional evidence that the ground state of benzonitrile is the valence ${ }^{2} \mathrm{~B}_{1}$ state. This work highlights the importance of balanced and accurate treatment of electron correlation and the need to consider nuclear motion.

\section{Acknowledgments:}

This work has been supported in Los Angeles by the Army Research Office through grant W911NF-16-1-0232 and in Munich by the German Research Foundation through grant JA2794/1-1 (Emmy Noether program). AIK is also a grateful recipient of the 2019 Simons Fellowship in Theoretical Physics, which supported her sabbatical stay in Germany.

Conflict of interests: A.I.K. is a member of directors and a part-owner of Q-Chem, Inc.

\section{Notes and references}

1 McGuire, B. A.; Burkhardt, A. M.; Kalenskii, S.; Shingledecker, C. N.; Remijan, A. J.; Herbst, E.; McCarthy, M. C. Detection of the aromatic molecule benzonitrile $c-\mathrm{C}_{6} \mathrm{H}_{5} \mathrm{CN}$ in the interstellar medium Science 2018, 359, 202-205.

2 Chiar, J. E.; Tielens, A. G. G. M.; Adamson, A. J.; Ricca, A. The structure, origin, and evolution of interstellar hydrocarbons grains Astrophys. J. 2013, 770, 78.

3 Kaiser, R. I.; Parker, D. S. N.; Mebel, A. M. Reaction dynamics in astrochemistry: Low-temperature pathways to polycyclic aromatic hydrocarbons in the interstellar medium Annu. Rev. Phys. Chem. 2015, 66, 43-67. 
4 Simons, J. Molecular anions J. Phys. Chem. A 2008, 112, 6401-6511.

5 Herbert, J. M. The quantum chemistry of loosely-bound electrons Rev. Comp. Chem. 2015, 28, 391-517.

6 Jordan, K.D.; Wang, F. Theory of dipole-bound anions Annu. Rev. Phys. Chem. 2003, 54, 367-396.

7 Gutowski, M.; Jordan, K.D.; Skurski, P. Electronic structure of dipole-bound anions J. Phys. Chem. A 1998, 102, 2624-2633.

8 Fortenberry, R.; Crawford, T. D. Theoretical prediction of new dipole-bound singlet states for anions of interstellar interest J. Chem. Phys. 2011, 134, 154304.

9 Lykke, K. R.; Mead, R. D.; Lineberger, W. C. Observation of dipole-bound states of negative ions Phys. Rev. Lett. 1984, 52, 2221-2224.

10 McCarthy, M. C.; Gottlieb, C. A.; Gupta, H.; Thaddeus, P. Laboratory and astronomical identification of the negative molecular ion $\mathrm{C}_{6} \mathrm{H}^{-}$Astrophys. J. Lett. 2006, 652, L141.

11 Brünken, S.; Gupta, H.; Gottlieb, C. A.; McCarthy, M. C.; Thaddeus, P. Detection of the carbon chain negative ion $\mathrm{C}_{8} \mathrm{H}^{-}$ in TMC-1 Astrophys. J. Lett. 2007, 664, L43.

12 Thaddeus, P.; Gottlieb, C. A.; Gupta, H.; Brünken, S.; McCarthy, M. C.; Agúndez, M.; Guelin, M.; Cernicharo, J. Laboratory and astronomical detection of the negative molecular ion $\mathrm{C}_{3} \mathrm{~N}^{-}$Astrophys. J. 2008, 677, 1132.

13 Cernicharo, J.; Guélin, M.; Agúndez, M.; Kawaguchi, K.; McCarthy, M.; Thaddeus, P. Astronomical detection of $\mathrm{C}_{4} \mathrm{H}^{-}$, the second interstellar anion Astronomy \& Astrophysics 2007, 467, L37-L40.

14 Cernicharo, J.; Guélin, M.; Agundez, M.; McCarthy, M. C.; Thaddeus, $\mathrm{P}$. Detection of $\mathrm{C}_{5} \mathrm{~N}^{-}$and vibrationally excited $\mathrm{C}_{6} \mathrm{H}$ in IRC+10216 Astrophys. J. Lett. 2008, 688, L83.

15 Agúndez, M.; Cernicharo, J.; Guélin, M.; Kahane, C.; Roueff, E.; Kłos, J.; Aoiz, F.J.; Lique, F.; Marcelino, N.; Goicoechea, J. R.; González-Garcia, M.; Gottlieb, C.A.; McCarthy, M.C.; Thaddeus, P. Astronomical identification of $\mathrm{CN}^{-}$, the smallest observed molecular anion Astronomy \& Astrophysics 2010, 517, L2.

16 Millar, T. J.; Walsh, C.; Field, T. A. Negative ions in space Chem. Rev. 2017, 117, 1765-1795.

17 Graupner, K.; Field, T. A.; Saunders, G. C. Experimental evidence for radiative attachment in astrochemistry from electron attachment to NCCCCN Astrophys. J. Lett. 2008, 685, L95.

18 Sebastianelli, F.; Gianturco, F. A. Metastable anions of polyynes: Dynamics of fragmentation/stabilization in planetary atmospheres after electron attachment Eur. Phys. J. D 2012, 66, 41.

19 Sebastianelli, F.; Gianturco, F. A. Stabilizing dicyanoacetylene anions in planetary atmospheres: quantum dynamics of its transient negative ions Eur. Phys. J. D 2010, 59, 389-398.

20 Douguet, N.; dosSantos, S. F.; Raoult, M.; Dulieu, O.; Orel, A. E.; Kokoouline, V. Theoretical study of radiative electron attachment to $\mathrm{CN}, \mathrm{C}_{2} \mathrm{H}$, and $\mathrm{C}_{4} \mathrm{H}$ radicals J. Chem. Phys. 2015, 142, 234309.

21 Khamesian, M.; Douguet, N.; dos Santos, S. F.; Dulieu, O.;
Raoult, M.; Brigg, W. J.; Kokoouline, V. Formation of $\mathrm{CN}^{-}$, $\mathrm{C}_{3} \mathrm{~N}^{-}$, and $\mathrm{C}_{5} \mathrm{~N}^{-}$molecules by radiative electron attachment and their destruction by photodetachment Phys. Rev. Lett. 2016, 117, 123001.

22 Carelli, F.; Gianturco, F. A.; Wester, R.; Satta, M. Formation of cyanopolyyne anions in the interstellar medium: The possible role of permanent dipoles J. Chem. Phys. 2014, 141, 054302.

23 Skomorowski, W.; Gulania, S.; Krylov, A. I. Bound and continuum-embedded states of cyanopolyyne anions Phys. Chem. Chem. Phys. 2018, 20, 4805-4817.

24 Dixon, A. R.; Khuseynov, D.; Sanov, A. Benzonitrile: Electron affinity, excited states, and anion solvation J. Chem. Phys. 2015, 143, 134306.

25 Kirnosov, N.; Adamowicz, L. Configuration and energy landscape of the benzonitrile anion Chem. Phys. Lett. 2017, 676, 32-38.

26 Wentworth, W. E.; Kao, L. W.; Becker, R. S. Electron affinities of substituted aromatic compounds J. Phys. Chem. 1975, 79, 1161-1169.

27 Zlatkis, A.; Lee, C. K.; Wentworth, W. E.; Chen, E. C. M. Constant current linearization for determination of electron capture mechanisms Anal. Chem. 1983, 55, 1596-1599.

28 Burrow, P. D.; Howard, A. E.; Johnston, A. R.; Jordan, K. D. Temporary anion states of hydrogen cyanide, methyl cyanide, and methylene dicyanide, selected cyanoethylenes, benzonitrile, and tetracyanoquinodimethane J. Chem. Phys. 1992, 96, 7570-7578.

29 Krylov, A. I. Equation-of-motion coupled-cluster methods for open-shell and electronically excited species: The hitchhiker's guide to Fock space Annu. Rev. Phys. Chem. 2008, 59, 433462.

30 Sneskov, K.; Christiansen, O. Excited state coupled cluster methods WIREs: Comput. Mol. Sci. 2012, 2, 566-584.

31 Bartlett, R. J. Coupled-cluster theory and its equation-ofmotion extensions WIREs: Comput. Mol. Sci. 2012, 2, 126138.

32 Purvis, G. D.; Bartlett, R. J. A full coupled-cluster singles and doubles model: The inclusion of disconnected triples J. Chem. Phys. 1982, 76, 1910-1918.

33 Raghavachari, K.; Trucks, G.W .; Pople, J. A.; Head-Gordon, M. A fifth-order perturbation comparison of electron correlation theories Chem. Phys. Lett. 1989, 157, 479-483.

34 Emrich, K. An extension of the coupled-cluster formalism to excited states (I) Nucl. Phys. 1981, A351, 379-396.

35 Sekino, H.; Bartlett, R. J. A linear response, coupled-cluster theory for excitation energy Int. J. Quant. Chem. 1984, 26, 255-265.

36 Koch, H.; Jørgensen, P. Coupled cluster response functions $J$. Chem. Phys. 1990, 93, 3333-3344.

37 Koch, H.; Jensen, H.J.Aa.; Jørgensen, P.; Helgaker, T. Excitation energies from the coupled clusters singles and doubles linear response functions (CCSDLR). Applications to $\mathrm{Be}, \mathrm{CH}^{+}$, CO, and $\mathrm{H}_{2} \mathrm{O}$ J. Chem. Phys. 1990, 93, 3345-3350.

38 Stanton, J. F.; Bartlett, R. J. The equation of motion coupled- 
cluster method. A systematic biorthogonal approach to molecular excitation energies, transition probabilities, and excited state properties J. Chem. Phys. 1993, 98, 7029-7039.

39 Nooijen, M.; Bartlett, R. J. Description of core-excitation spectra by the open-shell electron-attachment equation-of-motion coupled cluster method J. Chem. Phys. 1995, 102, 67356756.

40 Levchenko, S. V.; Krylov, A. I. Equation-of-motion spin-flip coupled-cluster model with single and double substitutions: Theory and application to cyclobutadiene J. Chem. Phys. 2004, 120, 175-185.

41 Krylov, A. I. In Reviews in Comp. Chem.; Parrill, A.L., Lipkowitz, K.B., Eds., Vol. 30; J. Wiley \& Sons, 2017; p. 151.

42 Nooijen, M.; Bartlett, R. J. Equation of motion coupled cluster method for electron attachment J. Chem. Phys. 1995, 102, 3629-3647.

43 Matthews, D. A.; Stanton, J. F. A new approach to approximate equation-of-motion coupled cluster with triple excitations J. Chem. Phys. 2016, 145, 124102.

44 Jagau, T. C. Non-iterative triple excitations in equation-ofmotion coupled-cluster theory for electron attachment with applications to bound and temporary anions J. Chem. Phys. 2018, 148, 024104.

45 Oana, C. M.; Krylov, A. I. Dyson orbitals for ionization from the ground and electronically excited states within equationof-motion coupled-cluster formalism: Theory, implementation, and examples J. Chem. Phys. 2007, 127, 234106-14.

46 Oana, C. M.; Krylov, A. I. Cross sections and photoelectron angular distributions in photodetachment from negative ions using equation-of-motion coupled-cluster Dyson orbitals $J$. Chem. Phys. 2009, 131, 124114-15.

47 Gozem, S.; Gunina, A. O.; Ichino, T.; Osborn, D. L.; Stanton, J. F.; Krylov, A. I. Photoelectron wave function in photoionization: Plane wave or Coulomb wave? J. Phys. Chem. Lett. 2015, 6, 4532-4540.

48 Ortiz, J.V. Toward an exact one-electron picture of chemical bonding Adv. Quantum Chem. 1999, 35, 33-52.

49 Jagau, T.-C.; Krylov, A. I. Characterizing metastable states beyond energies and lifetimes: Dyson orbitals and transition dipole moments J. Chem. Phys. 2016, 144, 054113.

50 Epifanovsky, E.; Zuev, D.; Feng, X.; Khistyaev, K.; Shao, Y.; Krylov, A. I. General implementation of resolution-of-identity and Cholesky representations of electron-repulsion integrals within coupled-cluster and equation-of-motion methods: The- ory and benchmarks J. Chem. Phys. 2013, 139, 134105.

51 Feng, X.; Epifanovsky, E.; Gauss, J.; Krylov, A. I. Implementation of analytic gradients for CCSD and EOM-CCSD using Cholesky decomposition of the electron-repulsion integrals and their derivatives: Theory and benchmarks 2019, 151, 014110.

52 ezSpectrum. Mozhayskiy, V. A.; Krylov, A. I.; ezSpectrum, http://iopenshell.usc.edu/downloads/.

53 ezDyson user's manual. Gozem, S.; Krylov, A. I. 2015; ezDyson, http://iopenshell.usc.edu/downloads/.

54 Zuev, D.; Jagau, T.-C.; Bravaya, K. B.; Epifanovsky, E.; Shao, Y.; Sundstrom, E.; Head-Gordon, M.; Krylov, A. I. Complex absorbing potentials within EOM-CC family of methods: Theory, implementation, and benchmarks J. Chem. Phys. 2014, 141, 024102.

55 Jagau, T.-C.; Zuev, D.; Bravaya, K. B.; Epifanovsky, E.; Krylov, A. I. A fresh look at resonances and complex absorbing potentials: Density matrix based approach J. Phys. Chem. Lett. 2014, 5, 310-315.

56 Jagau, T.-C.; Krylov, A. I. Complex absorbing potential equation-of-motion coupled-cluster method yields smooth and internally consistent potential energy surfaces and lifetimes for molecular resonances J. Phys. Chem. Lett. 2014, 5, 3078-3085.

57 Jagau, T.-C.; Dao, D. B.; Holtgrewe, N. S.; Krylov, A. I.; Mabbs, R. Same but different: Dipole-stabilized shape resonances in $\mathrm{CuF}^{-}$and $\mathrm{AgF}^{-}$J. Phys. Chem. Lett. 2015, 6, 27862793.

58 Shao, Y.; Gan, Z.; Epifanovsky, E.; Gilbert, A.T.B.; Wormit, M.; Kussmann, J.; Lange, A.W.; Behn, A.; Deng, J.; Feng, X., et al. Advances in molecular quantum chemistry contained in the Q-Chem 4 program package Mol. Phys. 2015, 113, 184-215.

59 Krylov, A. I.; Gill, P. M. W. Q-Chem: An engine for innovation WIREs: Comput. Mol. Sci. 2013, 3, 317-326.

60 Mulliken, R.S. Report on notation for the spectra of polyatomic molecules J. Chem. Phys. 1955, 23, 1997-2011.

61 Desfrancois, C.; Periquet, V.; Bouteiller, Y.; Schermann, J. P. Valence and dipole binding of electrons to uracil J. Chem. Phys. 1998, 102, 1274-1278.

62 Sommerfeld, T. Intramolecular electron transfer from dipolebound to valence orbitals: Uracil and 5-chlorouracil J. Phys. Chem. A 2004, 108, 9150-9154.

63 J. H. Hendricks, S. A. Lyapustina, H. L. de Clercq; Bowen, K. H. The dipole bound-to-covalent anion transformation in uracil J. Chem. Phys. 1998, 108, 8-11. 